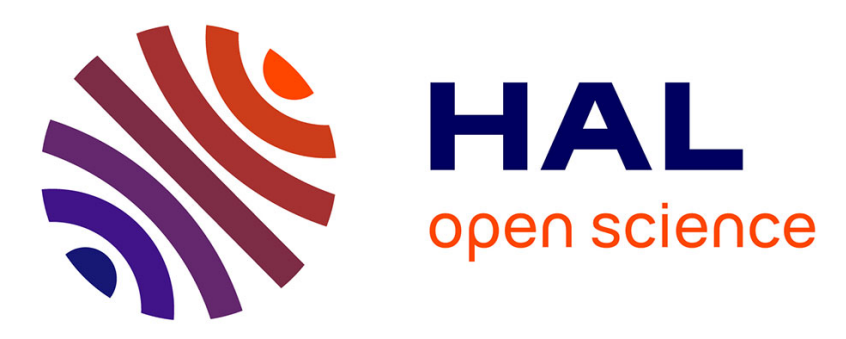

\title{
The decay of magnetohydrodynamic turbulence in a confined domain
}

\author{
Salah Neffaa, Wouter J.T. Bos, Kai Schneider
}

\section{To cite this version:}

Salah Neffaa, Wouter J.T. Bos, Kai Schneider. The decay of magnetohydrodynamic turbulence in a confined domain. Physics of Plasmas, 2008, 15, pp.092304. 10.1063/1.2975347 . hal-00444989

\author{
HAL Id: hal-00444989 \\ https://hal.science/hal-00444989
}

Submitted on 20 Jun 2012

HAL is a multi-disciplinary open access archive for the deposit and dissemination of scientific research documents, whether they are published or not. The documents may come from teaching and research institutions in France or abroad, or from public or private research centers.
L'archive ouverte pluridisciplinaire HAL, est destinée au dépôt et à la diffusion de documents scientifiques de niveau recherche, publiés ou non, émanant des établissements d'enseignement et de recherche français ou étrangers, des laboratoires publics ou privés. 


\title{
The decay of magnetohydrodynamic turbulence in a confined domain
}

\author{
Salah Neffaa, ${ }^{1}$ Wouter J. T. Bos, ${ }^{1,2}$ and Kai Schneider ${ }^{1}$ \\ ${ }^{1}$ M2P2-CNRS and CMI, Université de Provence, 39 Rue Frédéric Joliot-Curie, \\ 13453 Marseille Cedex 13, France \\ ${ }^{2}$ LMFA, UMR CNRS 5509, Ecole Centrale de Lyon-Université Claude Bernard Lyon 1-INSA de Lyon, \\ 69134 Ecully Cedex, France
}

(Received 29 February 2008; accepted 24 June 2008; published online 15 September 2008)

\begin{abstract}
The effect of nonperiodic boundary conditions on decaying two-dimensional magnetohydrodynamic turbulence is investigated. A circular domain with no-slip boundary conditions for the velocity is considered and where the normal component of the magnetic field vanishes at the wall. Different flow regimes are obtained by starting from random initial velocity and magnetic fields with varying integral quantities. These regimes, equivalent to the ones observed by Ting, Matthaeus, and Montgomery [Phys. Fluids 29, 3261 (1986)] in periodic domains, are found to subsist in confined domains. The effect of solid boundaries on the energy decay and alignment properties is examined. The final states are characterized by functional relationships between velocity and magnetic field. (C) 2008 American Institute of Physics. [DOI: 10.1063/1.2975347]
\end{abstract}

\section{INTRODUCTION}

The influence of initial conditions on decaying magnetohydrodynamic (MHD) turbulence received considerable interest in the 1980 s, because of its relevance to explain solarwind data. ${ }^{1-3}$ Indeed, in magnetohydrodynamics the behavior of decaying turbulent flow depends strongly on the initial conditions, and different initial values and ratios of integral quantities can lead to a wide variety of distinct behaviors. The first systematic study of the different possible types of decay was performed by Ting, Matthaeus, and Montgomery, ${ }^{4}$ who identified four classes of possible decay behavior, corresponding roughly to a magnetically dominated, a hydrodynamically dominated, a magnetically hydrodynamically equipartitioned and an erratic transition regime. Their study considered the two-dimensional case, which is not only relevant in applications in which an externally imposed field renders the flows quasi two-dimensional, but also from a general physical understanding of MHD turbulence, which behaves quite similar in two and three dimensions, due to the equivalent role of the ideal invariants. ${ }^{5}$

Whereas the influence of the initial conditions on decaying MHD turbulence has been studied and understood to some extend, studies on the effect of boundary conditions have been limited to low resolutions, ${ }^{6-8}$ imposed by the numerical methods used to account for boundaries. Even though these investigations highlighted interesting physics, higher resolution simulations are needed to obtain a better understanding of wall-bounded MHD, which plays a dominant role in geophysical flows in the core of planets such as the Earth and industrial processes involving liquid metals. For the hydrodynamic case it was found that boundary conditions have a significant influence on two-dimensional turbulence. ${ }^{9}$ In contrast to the periodic domain, where generally a long lasting state is found with a functional sinhrelationship between the vorticity and the stream function, ${ }^{10}$ corresponding to two counter-rotating vortices, in bounded domains with no-slip wall conditions different final states are observed depending on the geometry. ${ }^{11,12}$

In the present work we propose an extension of the volume penalization method ${ }^{13}$ to two-dimensional MHD to compute decaying flows in bounded domains using an efficient Fourier pseudospectral method. We address the following questions: What is the influence of confinement by fixed solid boundaries on decaying two-dimensional MHD turbulence? Do the four regimes found by Ting et al. ${ }^{4}$ continue to exist in the presence of boundaries? What are the final (viscously decaying) states?

\section{GOVERNING EQUATIONS AND NUMERICAL METHOD}

We consider resistive MHD, formulated in usual dimensionless variables $\mathbf{u}=(u, v)$ and $\mathbf{B}=\left(B_{x}, B_{y}\right)$ which are, respectively, the velocity and the magnetic field. The flow is considered to be two-dimensional, incompressible, and we assume the mass density to be constant. The governing equations are the following:

$$
\begin{aligned}
& \frac{\partial \mathbf{u}}{\partial t}+\mathbf{u} \cdot \nabla \mathbf{u}=-\nabla p+\mathbf{j} \times \mathbf{B}+\nu \nabla^{2} \mathbf{u}-\frac{1}{\epsilon} \chi\left(\mathbf{u}-\mathbf{u}_{0}\right), \\
& \frac{\partial \mathbf{B}}{\partial t}=\nabla \times(\mathbf{u} \times \mathbf{B})+\eta \nabla^{2} \mathbf{B}-\frac{1}{\epsilon} \chi\left(\mathbf{B}-\mathbf{B}_{0}\right), \\
& \nabla \cdot \mathbf{u}=0, \quad \nabla \cdot \mathbf{B}=0 .
\end{aligned}
$$

Here $\nu$ and $\eta$ are, respectively, the kinematic viscosity and the magnetic diffusivity. $\omega \mathbf{e}_{z}=\nabla \times \mathbf{u}$ is the vorticity, $\mathbf{j}=j \mathbf{e}_{z}=\nabla \times \mathbf{B}$ is the current density. Furthermore we define the vector potential $\mathbf{a}=a \mathbf{e}_{z}$ as $\mathbf{B}=\nabla \times \mathbf{a}$ and the stream function $\psi$ as $\mathbf{u}=\nabla^{\perp} \psi=(-\partial \psi / \partial y, \partial \psi / \partial x)$. An originality in our approach is the way in which the boundary conditions are imposed: we use volume (or surface in 2D) penalization ${ }^{13,14}$ to include the boundary conditions. This method has the advantage that arbitrary basis functions can be used. In our case 


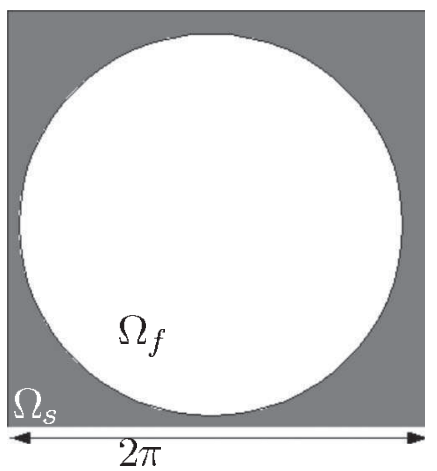

FIG. 1. The computational domain is a square box of size $2 \pi$. The fluid domain $\Omega_{f}$ is a circular container with radius $r=\frac{19}{20} \pi$, surrounded by the solid domain $\Omega_{s}$.

a Fourier pseudospectral code is employed. The advantage with respect to a method based on a decomposition in terms of Chandrasekhar-Kendall eigenfunctions ${ }^{6-8}$ is, that fast Fourier transforms can be used, allowing for high resolution computations of low computational cost. Also, its application to three-dimensional flows is conceptually straightforward and will be addressed in a future work. The additional terms on the right-hand side of Eqs. (1) and (2) correspond to this penalization method. The quantities $\mathbf{u}_{\mathbf{0}}$ and $\mathbf{B}_{\mathbf{0}}$ correspond to the values imposed in the solid part of the numerical domain $\Omega_{s}$, illustrated in Fig. 1. Here we choose $\mathbf{u}_{\mathbf{0}}=\mathbf{0}$ and $\mathbf{B}_{\mathbf{0}}=\mathbf{B}_{\|}$ (where $\mathbf{B}_{\|}$is the tangential component of $\mathbf{B}$ at the wall), corresponding to vanishing velocity and no penetration of magnetic field into the solid domain which is hence considered as a perfect conductor, coated inside with a thin layer of insulant, which guarantees that the current density cannot penetrate into the solid. ${ }^{6}$ The mask function $\chi$ is equal to 0 inside $\Omega_{f}$ (where the penalization terms thereby disappear) and equal to 1 inside $\Omega_{s}$. The physical idea is to model the solid part as a porous medium whose permeability $\epsilon$ tends to zero. ${ }^{13,14}$ For $\epsilon \rightarrow 0$, where the obstacle is present, the velocity $\mathbf{u}$ tends to $\mathbf{u}_{\mathbf{0}}$ and the magnetic field $\mathbf{B}$ tends to $\mathbf{B}_{\mathbf{0}}$. The nature of the boundary condition for the velocity is thus noslip at the wall.

In the two-dimensional case it is convenient to take the curl of Eqs. (1) and (2) to obtain after simplification equations for the vorticity and current density. These are scalar valued equations which automatically satisfy the incompressibility conditions (3). The equations are then

$$
\frac{\partial \omega}{\partial t}=-\mathbf{u} \cdot \nabla \omega+\mathbf{B} \cdot \nabla j+\nu \nabla^{2} \omega-\frac{1}{\epsilon} \nabla \times\left[\chi\left(\mathbf{u}-\mathbf{u}_{0}\right)\right],
$$

$$
\frac{\partial j}{\partial t}=-\nabla^{2}(\mathbf{u} \times \mathbf{B})+\eta \nabla^{2} j-\frac{1}{\epsilon} \nabla \times\left[\chi\left(\mathbf{B}-\mathbf{B}_{0}\right)\right] .
$$

The equations are discretized with a classical Fourier pseudospectral method imposing periodic boundary conditions on the square domain of size $2 \pi$, using $512^{2}$ grid points. At each iteration the fields are dealiased by spherical truncation following the $2 / 3$ rule. The penalization parameter $\epsilon$, corresponding to the permeability of the solid domain, is taken equal to $10^{-3}$, a value validated by a systematic study of the sensitivity of the results to this parameter. ${ }^{14}$ The fluid viscosity $\nu$ and magnetic diffusivity $\eta$ were taken equal to $10^{-3}$, and the time step is equal to $5 \cdot 10^{-4}$. As the numerical scheme uses an explicit discretization of the penalization term, the time step is limited by the permeability $\epsilon$ due to stability reasons. Typically, using a Pentium 4 processor with $2 \mathrm{GHz}$ clock frequency, one time step integration requires $2.5 \mathrm{~s}$. The initial kinetic and magnetic Reynolds numbers are defined as $\operatorname{Re}=2 r \sqrt{2 E_{u}(t=0)} / \nu$ and $\operatorname{Re}_{m}=2 r \sqrt{2 E_{u}(t=0)} / \eta$, where $r$ is the radius of the domain, and $E_{u}$ is the kinetic energy.

\section{INITIAL CONDITIONS}

Both vorticity and current density fields are initialized with Gaussian random initial conditions. Their Fourier transforms $\hat{\omega}$ and $\hat{j}$, where $\hat{\omega}(\mathbf{k})=1 / 4 \pi^{2} \int \omega(\mathbf{x}) \mathbf{e}^{-l \mathbf{k} \cdot \mathbf{x}} d^{2} x$, are initialized with random phases and their amplitudes yield the energy spectra,

$$
E_{u}(k), E_{B}(k) \propto \frac{k}{\left[g+\left(k / k_{0}\right)\right]^{4}},
$$

with $k=|\mathbf{k}|$ and, where $g=0.98$ and $k_{0}=\frac{3}{4} \sqrt{2} \pi$. This energy spectrum follows a power law proportional to $k^{-3}$ at large wavenumbers and was chosen to compare with simulations performed in the periodic case. Both fields are statistically identical. The corresponding fields $\mathbf{u}$ and $\mathbf{B}$ are calculated from $\omega$ and $j$ using the Biot-Savart law.

For vanishing viscosity and resistivity, two-dimensional MHD has three conserved invariants. The total energy is $E$, defined as the sum of the kinetic energy $E_{u}$ and the magnetic energy $E_{B}$,

$$
E=E_{u}+E_{B}=\frac{1}{2} \int_{\Omega_{f}}\left(|\mathbf{u}|^{2}+|\mathbf{B}|^{2}\right) d^{2} x,
$$

$H_{c}$ is the cross helicity,

$$
H_{c}=\frac{1}{2} \int_{\Omega_{f}} \mathbf{u} \cdot \mathbf{B} d^{2} x,
$$

which measures the global correlations between $\mathbf{u}$ and $\mathbf{B}$, and $A$ is the integral of the squared vector potential,

$$
A=\frac{1}{2} \int_{\Omega_{f}} a^{2} d^{2} x .
$$

As was shown by Ting et $\mathrm{al}^{4}$ for periodic boundary conditions, the dynamics of decaying MHD turbulence depend strongly on the initial values of these invariants. Because of its interest for the present study we recall briefly the four distinct decay regimes discerned by Ting et al. ${ }^{4}$ depending on the initial values and ratios of the invariants. First, in the case of small initial $H_{c}$ and $E_{B}>E_{u}$, a magnetically dominated regime is obtained. Selective decay is observed in this regime which corresponds to a slower decay of $A$ relative to $E$. Second, in the case of vanishingly small initial magnetic energy, the Lorentz force acting in the vorticity equation can not become strong enough, so that the vector potential is advected like a passive scalar. Following Biskamp and 
TABLE I. Initial values of the four different regimes.

\begin{tabular}{lcccc}
\hline \hline & $E / A$ & $E_{u} / E_{B}$ & $H_{c}$ & $\operatorname{Re}$ \\
\hline Regime I & 16 & 0.3 & 0.012 & 3868 \\
Regime II & $3.4 \times 10^{5}$ & $1.9 \times 10^{4}$ & $3.5 \times 10^{-5}$ & 7920 \\
Regime III & 31 & 1.3 & 0.27 & 5176 \\
Regime IV & 16 & 1.0 & 0.045 & 5725 \\
\hline \hline
\end{tabular}

Welter, ${ }^{15}$ the magnetic field may however be amplified even if the initial ratio $E_{B} / E_{u}$ is very small, given that $\eta$ is sufficiently small. They found that $E_{u} / E_{B}<\mathrm{Re}_{m}$ is necessary such that the magnetic field can be intensified. This is a regime which essentially corresponds to the Navier-Stokes limit. Third, in the case of substantial initial cross-helicity, the turbulence tends towards an Alfvénic state in which $\mathbf{u}$ and $\mathbf{B}$ are aligned or antialigned and approximately equipartitioned. This process is called dynamic alignment and the ratio $E /\left|H_{c}\right|$ tends to 2 . This is a state free from nonlinear interactions, inhibiting cascade processes (even though this depletion of nonlinearity is rather slow with increasing cross-helicity ${ }^{3}$ ). The fourth and final regime is an erratic regime which might tend to different final states and which could be related to various competing subregions with unequal sign of cross-helicity. This regime can be found if the flow is initialized with small cross-helicity and comparable kinetic and magnetic energies.

Whether these regimes persist in the presence of solid boundaries is one of the main questions we want to answer in the present work. To obtain the desired initial conditions corresponding to the four regimes we proceed as follows:

Starting from random initial conditions in Fourier space, we renormalize $\mathbf{u}$ and $\mathbf{B}$ in physical space by varying the coefficient $\alpha$,

$$
\mathbf{u}^{*}=\frac{\alpha}{\sqrt{2 \mathbf{E}_{\mathbf{u}}}} \mathbf{u}, \quad \mathbf{B}^{*}=\frac{1}{\sqrt{2 \mathbf{E}_{\mathbf{B}}}} \mathbf{B} .
$$

This generally yields initial conditions with vanishingly small cross-helicity, and initial conditions for regimes I, II, and IV can hereby be created. In the case of regime III, a nonzero cross-helicity needs to be imposed. We achieve this by creating a random initial condition for $\mathbf{u}$ and a perpendicular field $\mathbf{u}_{\perp}$ by rotating $\mathbf{u}$ by $\pi / 2$. The magnetic field is then obtained by a linear combination of the two fields,

$$
\mathbf{B}^{*}=\beta \mathbf{u}+(1-\beta) \mathbf{u}_{\perp} .
$$

Hereby any given cross helicity can be imposed. Table I summarizes the initial values of $E / A, E_{u} / E_{B}$, and $H_{c}$ for the four different regimes, together with the Reynolds number.

\section{RESULTS AND DISCUSSION}

\section{A. Characterization of the different decay regimes}

In Fig. 2 we show the time evolution of several integral quantities for the four different sets of initial conditions. The main observation is that the four different regimes, discerned by Ting $e t ~ a l .{ }^{4}$ are robust enough to survive within a bounded domain. We now discuss the results in more detail.
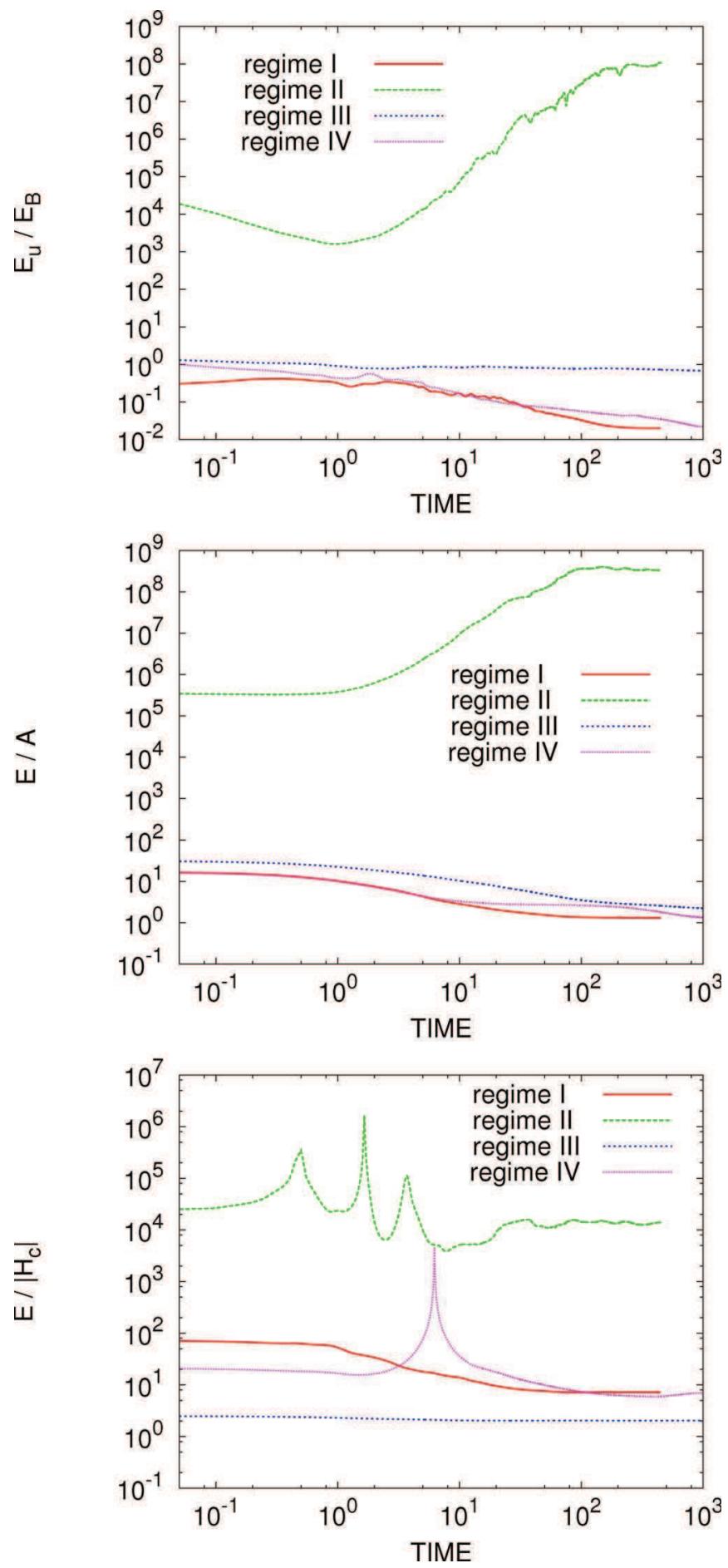

FIG. 2. (Color online) Time evolution of integral quantities in the bounded domain. Top: Ratio of kinetic and magnetic energy, $E_{u} / E_{B}$. Center: Ratio of total energy and integral of the squared vector potential, $E / A$. Bottom: Ratio of total energy and magnetic helicity, $E /\left|H_{c}\right|$.

Regarding the ratio of kinetic and magnetic energy (Fig. 2 , top), it is observed that in the absence of initial crosshelicity (cases I, II, and IV) the magnetic energy finally dominates, unless it is very small initially (Navier-Stokes limit). However, if the initial cross-helicity is initially large and $E_{u} / E_{B}$ is of order unity, the flow energy will remain approximately equipartitioned between the velocity and magnetic field. 
This picture is confirmed by the time evolution of the ratio $E / A$ (Fig. 2, center). In this representation it is however emphasized that in the Navier-Stokes limit (case II), the character of the magnetic field has changed; in the ideal system (vanishing viscosity and magnetic diffusivity), $A$ is a quantity that cascades towards the small wavenumbers. In a nonideal system an inverse cascade generally slows down the dissipation rate of the quantity. However, in the limit of small Lorentz force, the equations of the vorticity and vector potential become equivalent to the equations that describe a passive scalar advected by a two-dimensional velocity field. The passive scalar is a quantity which cascades towards higher wavenumbers, and the vector potential gets dissipated faster in this case than in the case where the Lorentz force is significant. This results in a rapid increase of the quantity $E / A$ in case II.

The ratio $E /\left|H_{c}\right|$ (Fig. 2, bottom) attains its minimum absolute value 2 for case III. This corresponds to dynamic alignment; the velocity field is equal in magnitude and perfectly aligned, or antialigned with the magnetic field. The erratic regime is clearly represented by case IV, in which the cross-helicity approaches a value close to zero. As we will see in the following, this is caused by different subregions with oppositely valued $H_{c}$.

For comparison we show in Fig. 3 the same quantities as in Fig. 2 for a periodic domain, starting from similar initial conditions and using the same numerical parameters as in the bounded case. It is observed that the trends are similar. In Fig. 3 (top) we see that in the periodic domain a more oscillatory behavior is observed for $E_{u} / E_{B}$ in regime I. This oscillatory behavior is related to energy exchange between the magnetic field and the velocity field by means of Alfvén waves. ${ }^{16}$ Whereas in a periodic domain these waves can freely propagate, in a bounded domain they might be more rapidly suppressed, explaining the less oscillatory behavior of $E_{u} / E_{B}$ in a bounded domain. Further research is needed to clarify this.

The quantity $E / H_{c}$ gives a measure for the dynamic alignment, which corresponds to measuring both the equipartitioning of energy and the alignment properties. If we are exclusively interested in the alignment properties, the relative cross helicity, which corresponds to the cosine of the angle $\theta$ between the velocity and magnetic field vector,

$$
\cos \theta=\frac{H_{c}}{\left(E_{u} E_{B}\right)^{1 / 2}},
$$

should be considered.

In Fig. $4, \cos \theta$ is plotted as a function of time. It can be observed that in cases I and III, the velocity field tends to a nearly aligned state. In cases II and IV, this quantity remains close to zero, however for a different reason. In case II, the alignment is small, because the vector potential is advected as a nearly passive scalar. In case IV the local alignment is large but different aligned or antialigned regions cancel out the contributions, yielding a net-global alignment close to 0 . A similar process was found for periodic boundary conditions. ${ }^{17,18}$ This can be observed in the corresponding
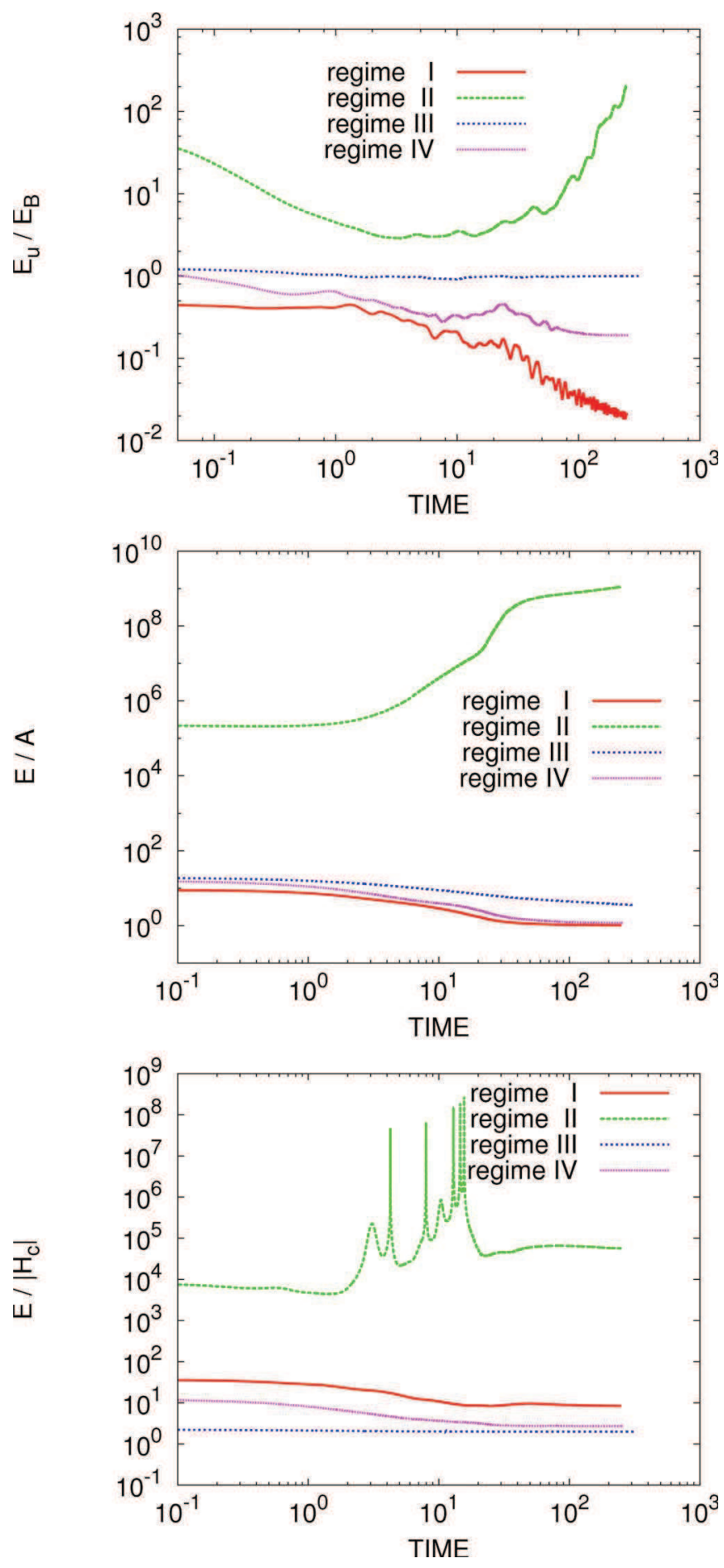

FIG. 3. (Color online) Time evolution of $E_{u} / E_{B}$ (top), $E / A$ (center), and $E /\left|H_{c}\right|$ (bottom) in a periodic domain, starting from similar initial conditions as in Fig. 2.

probability distribution function of $\cos \theta$ at $t=40$ and $t=450$, shown in Fig. 5. Nevertheless, for a long time $(t=1250)$ we observe an antialignment.

\section{B. Energy decay and visualizations}

The decay of total energy is shown in Fig. 6. At intermediate times, the energy in cases I and IV decays following a power law with exponents varying for the different sets of 


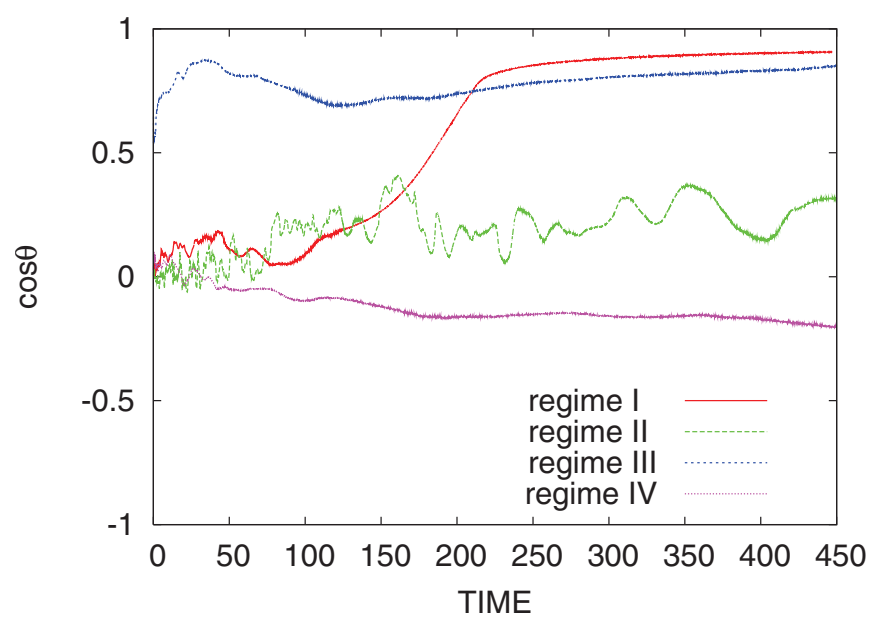

FIG. 4. (Color online) Time evolution of the average alignment $\cos \theta$, between the magnetic field and the velocity field.

initial conditions (Fig. 6, top). The exponents of these power laws are approximately -0.6 (dotted line) for regime I and -0.4 (solid line) for regime IV. It is seen that these power laws are observed only after an initial period of rapid decay. In the other cases no clear power law behavior can be identified. This can be compared to previous studies ${ }^{5,20}$ in which values around -0.75 and -1 were found for the decaying periodic case. In case III, in which dynamic alignment is observed, no clear power-law behavior is observed. In this case the nonlinear interactions are progressively damped by the alignment process, so that no self-similar period is observed in the energy decay. At late times (Fig. 6, bottom) all cases show an exponential viscous decay of the form $E \sim e^{-2 \alpha \nu t}$ with $\alpha=1.5$ in case I and $\alpha=2$ in cases II, III, and IV, a value related to the largest Stokes eigenmode of the circle $(\alpha=1.64)$, which contains most of the energy, as found in Ref. 11 for the hydrodynamical case.

Figures 7 and 8 show the vorticity and the current density field, respectively. For each of the cases I-IV, three typical time instants are visualized. These instants are $t=5$,

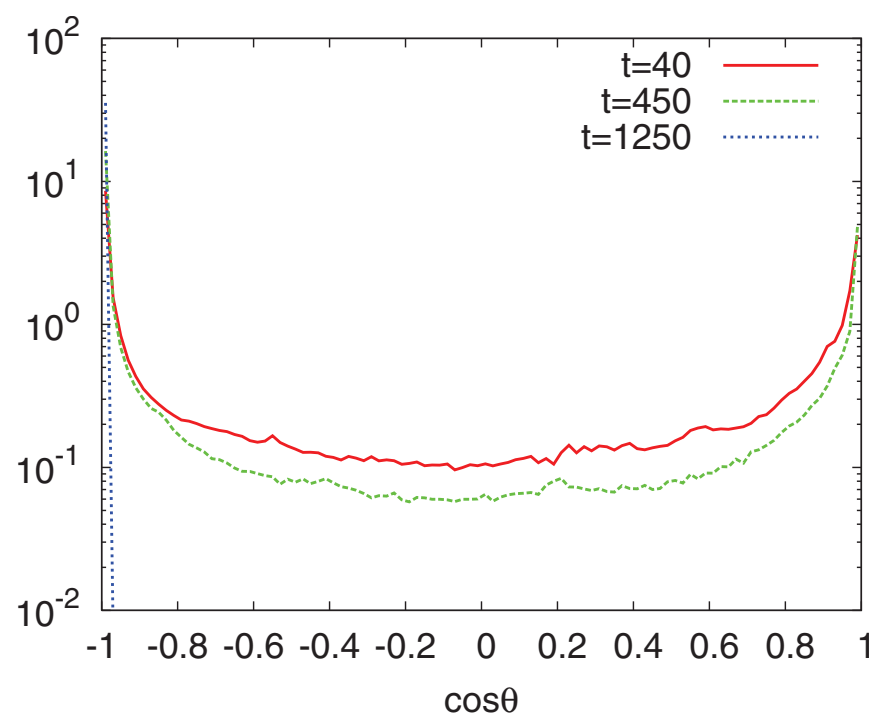

FIG. 5. (Color online) Probability density of $\cos \theta$ at $t=40, t=450$, and $t=1250$ in regime IV.
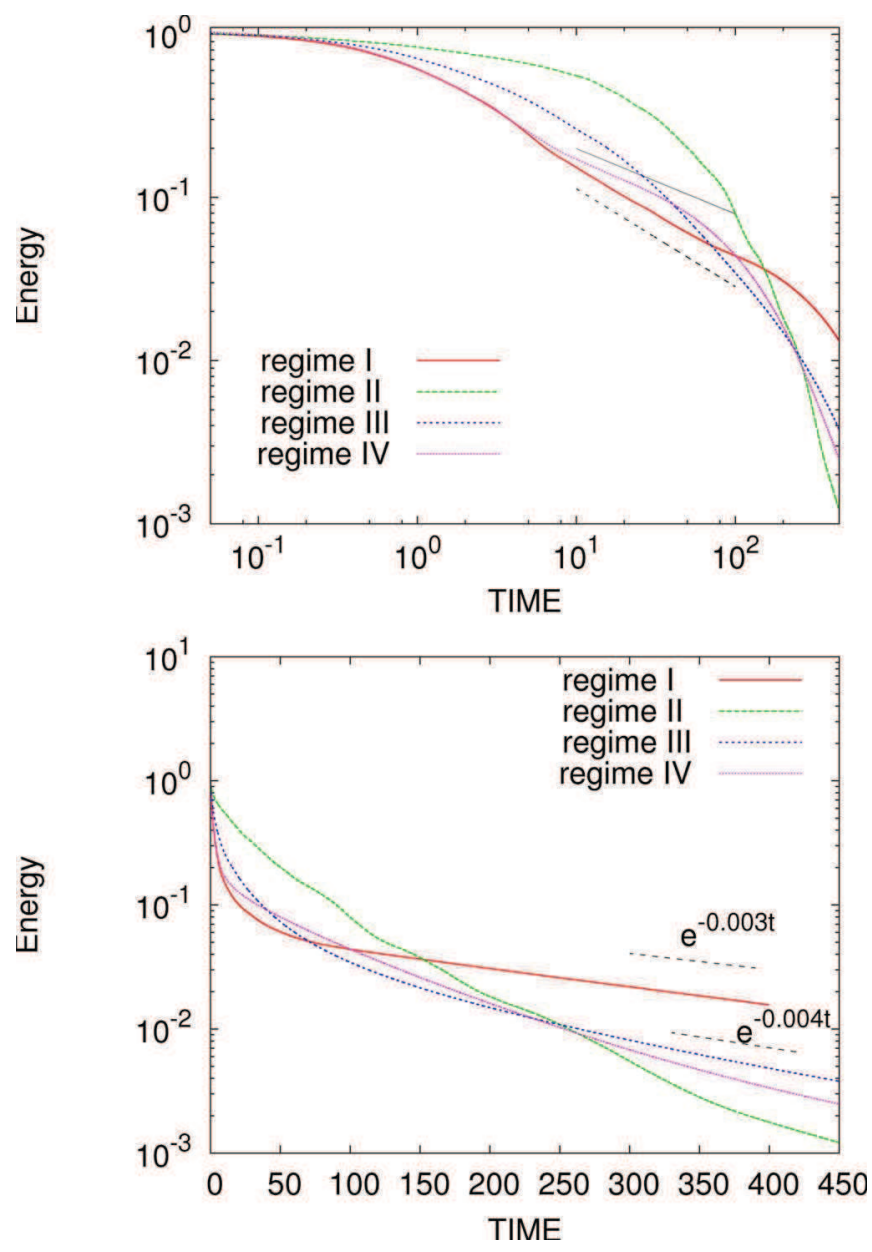

FIG. 6. (Color online) Time evolution of the total energy in log-log scale (top) and in log-lin scale (bottom). The solid line (top) corresponds to $t^{-0.4}$ and the dotted line (top) corresponds to $t^{-0.6}$.

showing the self-organization of the flow at early times, $t=40$, when nonlinear processes are dominating and $t=450$ (regime I) and $t=1250$ (regimes II, III, and IV), corresponding to the final, viscously decaying state.

One flagrant feature of the visualizations is the local alignment of the magnetic and velocity field. Indeed in most regimes the vorticity and current density fields are rather similar. We also observe the coincidence of the maxima of $\omega$ and of $j$ which may have some effect on the stabilization of vorticity and current filaments. In case I an almost perfect axisymmetrical state is achieved at $t=450$. Case II is the only case in which the formation of circular vortices is well pronounced, leading to a roll up of the current sheets. Apparently in the other regimes the Lorentz force suppresses the generation of circular vortices. Case III shows almost identical magnetic and velocity fields, as expected in this case of dynamic alignment, in which $\mathbf{u}$ and $\mathbf{B}$ are aligned (or antialigned) and in which kinetic and magnetic energies are in equipartition. Case IV is a typical example of the erratic regime; at the intermediate time, four dominant flow structures are observed, with both positive and negative crosshelicity. Locally the flow is close to an aligned or antialigned state, but globally the cross-helicity is weak because the different regions with opposite contributions cancel each other out. 


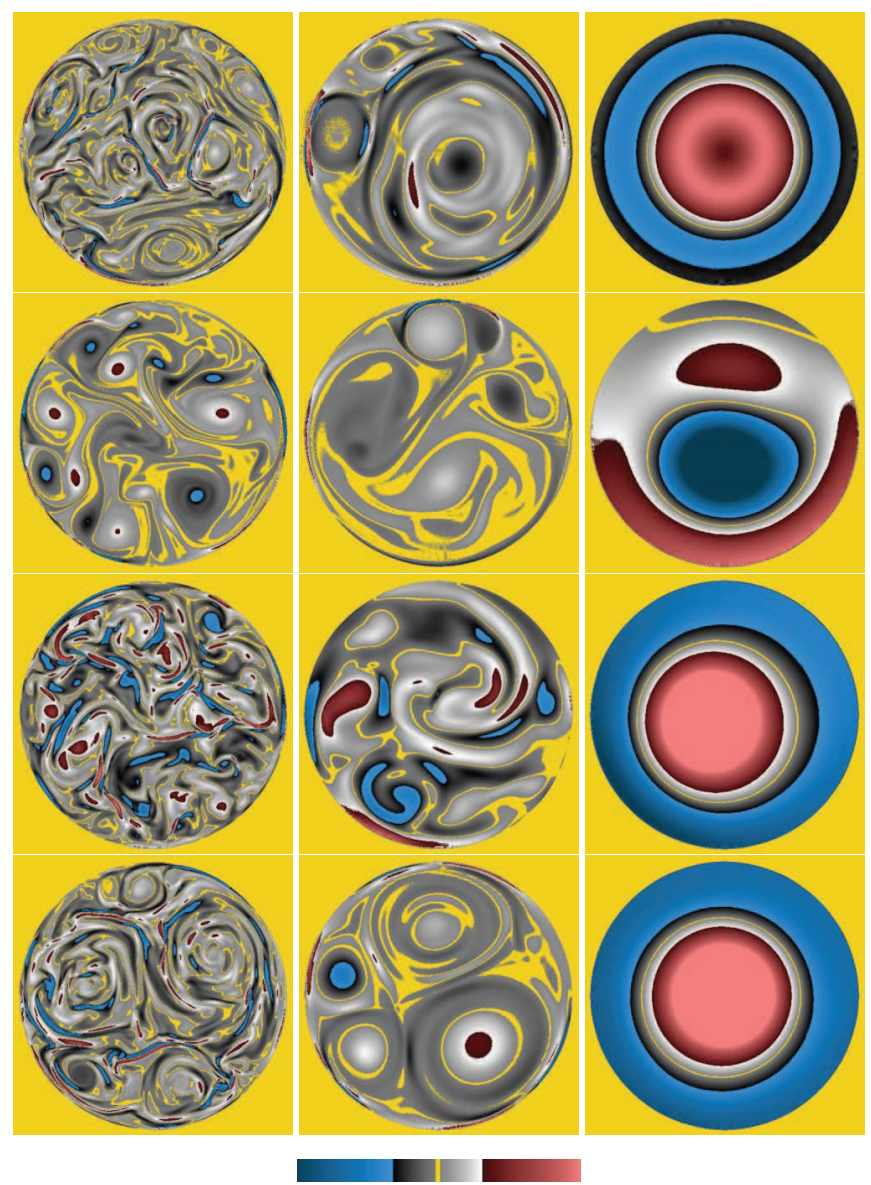

FIG. 7. (Color online) Vorticity at different instants in the circular domain. From top to bottom: Regime I, regime II, regime III, and regime IV. From left to right: $t=5, t=40$ and in the last column the time corresponds to $t$ $=450$ for regime I and $t=1250$ for regimes II, III, and IV.

\section{Final states}

Supplementary information on the final states is given by scatter-plots. It was shown by Joyce and Montgomery ${ }^{10}$ that in hydrodynamic unbounded two-dimensional flows a long lasting final state is reached, depleted from nonlinearity. This state is characterized by a functional relation between the vorticity and the stream function of the form $\omega \sim \sinh (\psi)$. That a functional relation leads to a state, depleted from nonlinearity is easily shown from the equation for the vorticity,

$$
\left(\partial_{t}-\nu \Delta\right) \omega=[\omega, \psi],
$$

with the Poisson bracket defined as $[a, b]=(\partial a / \partial x)(\partial b / \partial y)$ $-(\partial a / \partial y)(\partial b / \partial x)$. A functional relation $\omega=F(\psi)$ leads to a vanishing Poisson bracket. If we consider now the equations for incompressible MHD,

$$
\begin{aligned}
& \left(\partial_{t}-\nu \Delta\right) \omega=[\omega, \psi]-[a, j], \\
& \left(\partial_{t}-\eta \Delta\right) a=[a, \psi],
\end{aligned}
$$

we see that two nonlinearities play a role: $[\omega, \psi]$ and $[a, j]$. The term $[a, \psi]$ can be considered as a pseudononlinearity if $\psi$ is regarded as given. Although important theoretical progress has been made in the comprehension of final states ${ }^{19}$ no analytical nontrivial solution is presently known

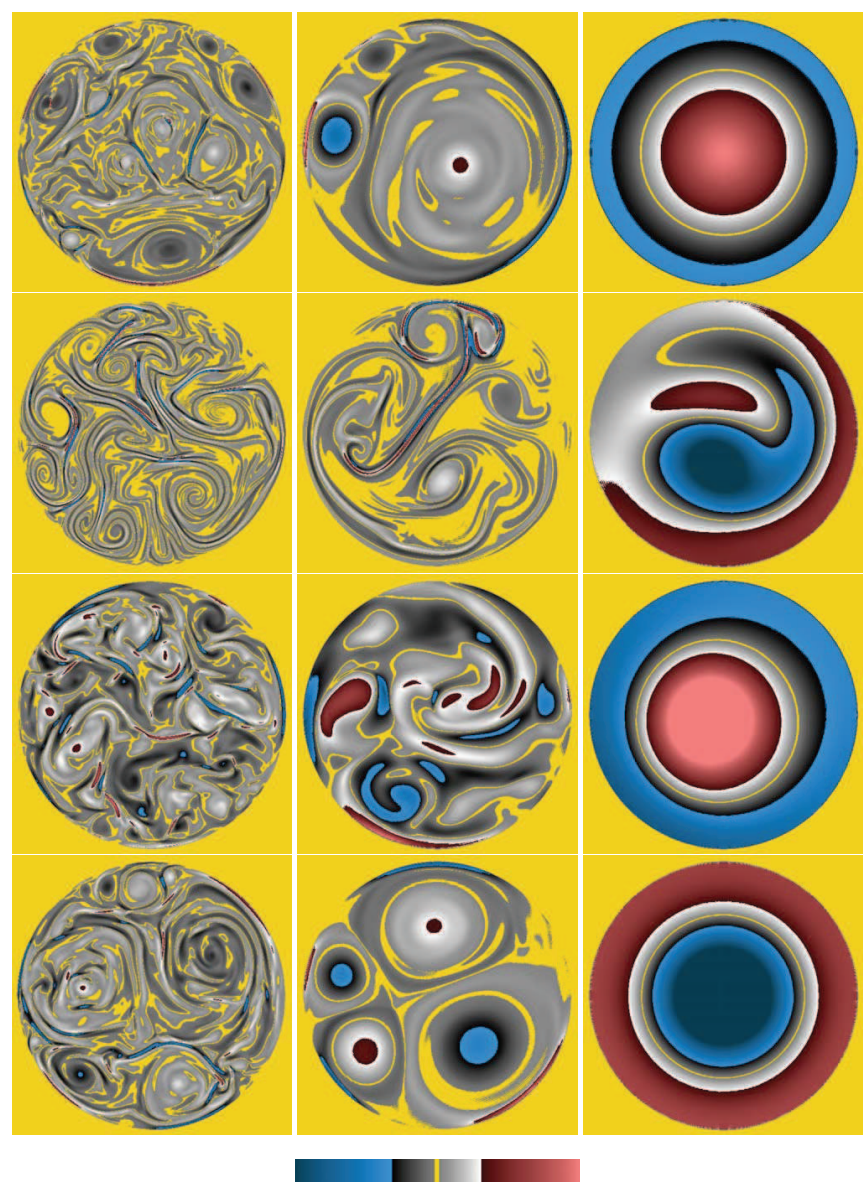

FIG. 8. (Color online) Current density at different instants in the circular domain. From top to bottom: Regime I, regime II, regime III, and regime IV. From left to right: $t=5, t=40$ and in the last column the time corresponds to $t=450$ for regime I and $t=1250$ for regimes II, III, and IV.

for the case of decaying MHD turbulence. It was however shown in Kinney et $a l^{20}$ that close to functional relations do exist in homogeneous two-dimensional MHD turbulence. In Fig. 9 we show for the cases I-IV these scatter plots corresponding to the three nonlinearities.

In case I we see a well defined nonlinear functional relation $\omega(\psi)$. Clearly, we have a nontrivial final state. The evolution of $\omega-\psi$ as a function of time in this case is shown in Fig. 10. It can be noted here that a similarly shaped scatter plot was found in close to two-dimensional hydrodynamic experiments in a circular tank. ${ }^{21}$ The plot $a$ versus $j$ shows a straight line (Fig. 9), which corresponds to a vanishing Lorentz-force; the magnetic field does not interact with the velocity field at this final period of decay. The plot $a$ versus $\psi$ also shows a clear functional relation. In case II, the scatter plots do not show such clear functional relations which is due to the fact that the flow is not yet sufficiently relaxed. The plot $\omega$ versus $\psi$ is perhaps closest to a functional relation. In case III we see, as expected, a vanishing nonlinearity; for dynamic alignment it can be shown that nonlinearities vanish in the perfectly aligned case, when the equations are stated in Elsässer variables (see, for example, Ref. 3). In case IV it is expected that eventually the same behavior is observed as in case I. If the initial Reynolds number is initially too low this behavior will however not be observed. 

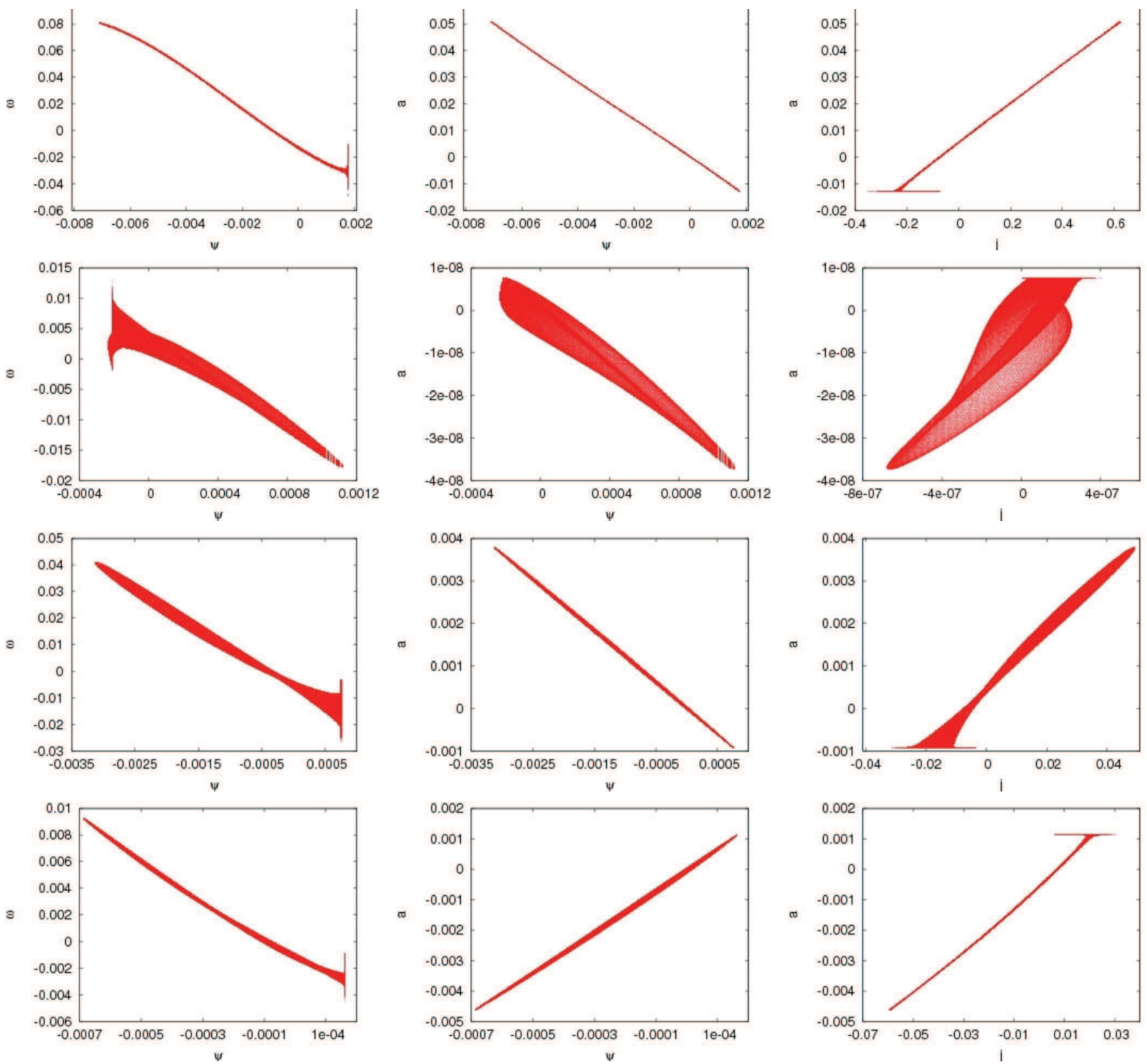

FIG. 9. (Color online) Scatter plots of (from left to right) $\omega$ vs $\psi, a$ vs $\psi$, and $a$ vs $j$ for regimes (from top to bottom) I, II, III, and IV at the latest time instant $t=450$ for regime I and $t=1250$ for regimes II, III, and IV.

Preliminary computations were performed at lower resolution, which showed that nontrivial final states are only observed if the initial Reynolds number is sufficiently high. Otherwise linear relations are obtained for all different scatter plots.

\section{CONCLUSION}

We have investigated the influence of nonperiodic boundary conditions on decaying two-dimensional magnetohydrodynamic turbulence. The use of a penalization method in combination with a classical Fourier pseudospectral method allows for efficient resolution of MHD flows in bounded domains. A main result is the observation of the robustness of the four different regimes discerned by Ting et $a l .{ }^{4}$ The same trends are found as in their pioneering work,

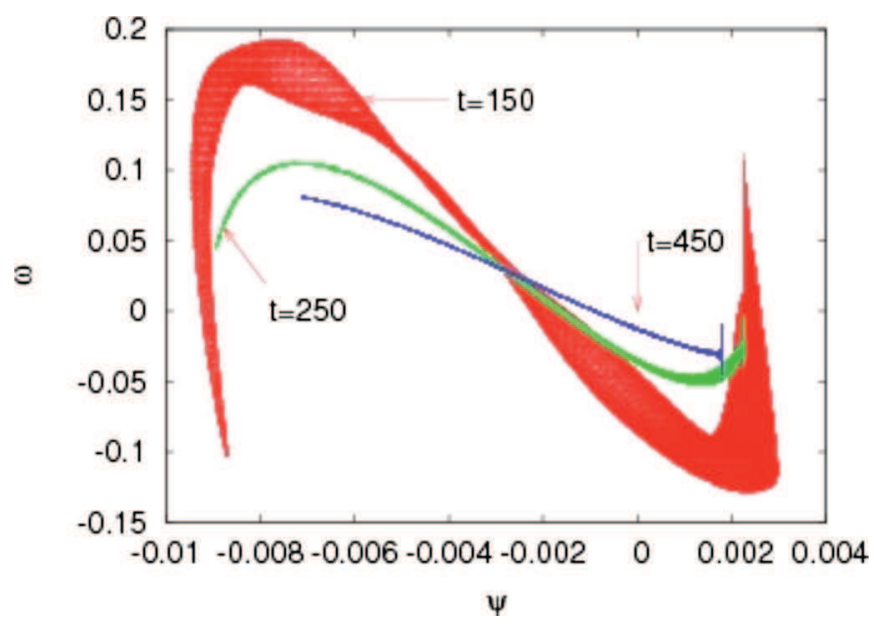

FIG. 10. (Color online) Scatter plot of $\omega$ vs $\psi$ at three different instants in regime $\mathrm{I}$. 
depending on the initial values of the kinetic energy, magnetic energy, vector potential, and cross-helicity.

A detailed description was given of the relaxation process which leads to the final states. In the case of a magnetically dominant, cross-helicity free case, a clear nontrivial functional relation $\omega(\psi)$ was observed. Functional relationships were also observed in regimes III and IV, while in regime II this functional relation was less clear.

Future work will address the influence of other types of boundary conditions for the magnetic field and also other geometries will be studied.

Note added in proof. The numerical method used in the present work does not impose a zero value of $a$ and $\psi$ at the wall of the fluid domain. If one wants to impose this, a constant value has to be substracted from $a$ and $\psi$ at every point in the fluid domain. Note that this will also change the integral value $A$.

\section{ACKNOWLEDGMENTS}

We gratefully acknowledge Professor David Montgomery for his valuable remarks, and Diego del-Castillo-Negrete and Florin Spineanu for useful discussions. We acknowledge financial support from the Agence Nationale de la Recherche, project "M2TFP."
${ }^{1}$ M. Dobrowolny, A. Mangeney, and P. Veltri, Phys. Rev. Lett. 45, 144 (1980).

${ }^{2}$ R. Grappin, U. Frisch, J. Leorat, and A. Pouquet, Astron. Astrophys. 105, 6 (1982).

${ }^{3}$ W. H. Matthaeus, M. L. Goldstein, and D. C. Montgomery, Phys. Rev. Lett. 51, 1484 (1983).

${ }^{4}$ A. C. Ting, W. H. Matthaeus, and D. C. Montgomery, Phys. Fluids 29, 3261 (1986).

${ }^{5}$ D. Biskamp, and E. Schwarz, Phys. Plasmas 8, 3282 (2001).

${ }^{6}$ P. D. Mininni and D. C. Montgomery, Phys. Fluids 18, 116602 (2006).

${ }^{7}$ P. D. Mininni, D. C. Montgomery, and L. Turner, New J. Phys. 9, 303 (2007).

${ }^{8}$ X. Shan and D. C. Montgomery, Phys. Rev. Lett. 73, 1624 (1994).

${ }^{9}$ K. Schneider and M. Farge, Phys. Rev. Lett. 95, 244502 (2005).

${ }^{10}$ G. Joyce and D. Montgomery, J. Plasma Phys. 10, 107 (1973).

${ }^{11}$ K. Schneider and M. Farge, Physica D 237, 2228 (2008).

${ }^{12} \mathrm{~S}$. Li and D. Montgomery, Theor. Comput. Fluid Dyn. 9, 167 (1997).

${ }^{13}$ P. Angot, C. H. Bruneau, and P. Fabrie, Numer. Math. 81, 497 (1999).

${ }^{14}$ K. Schneider, Comput. Fluids 34, 1223 (2005).

${ }^{15}$ D. Biskamp and H. Welter, Phys. Fluids B 2, 1787 (1990).

${ }^{16}$ A. Pouquet, P. L. Sulem, and M. Meneguzzi, Phys. Fluids 31, 2635 (1988).

${ }^{17}$ W. H. Matthaeus, A. Pouquet, P. D. Mininni, P. Dmitruk, and B. Breech, Phys. Rev. Lett. 100, 085003 (2008).

${ }^{18}$ S. Servidio, W. H. Matthaeus, and P. Dmitruk, Phys. Rev. Lett. 100, 095005 (2008).

${ }^{19}$ F. Spineanu and M. Vlad, Phys. Rev. E 67, 046309 (2003).

${ }^{20}$ R. Kinney, J. C. McWilliams, and T. Tajima, Phys. Plasmas 2, 3623 (1995).

${ }^{21}$ F. de Rooij, P. F. Linden, and S. B. Dalziel, J. Fluid Mech. 383, 249 (1999). 